\title{
EXPANSION OF CONVEX HYPERSURFACES BY NONHOMOGENEOUS FUNCTIONS OF CURVATURE*
}

\author{
BENNETT $\mathrm{CHOW}^{\dagger}$ AND DONG-HO TSAI ${ }^{\ddagger}$
}

1. Introduction. This paper is a sequel to our previous ones, Chow-Liou-Tsai [CLT] and Chow-Tsai [CT2], where we considered the expansion of a smooth closed uniformly convex hypersurface $M_{0}$ in Euclidean space along its outward normal vector direction with speed a function of the inverse of the harmonic mean curvature and the Gauss curvature respectively. Here we shall study the more general case concerning the expansion of a closed uniformly convex hypersurface $M_{0}$ of dimension $n$ in Euclidean space $\mathbb{R}^{n+1}$ with the speed $f$ a nonhomogeneous positive function of the principal radii. Needless to say, there have been many important works on the homogeneous flows. We refer the readers to the introductory remarks in the papers by Chow-Tsai [CT1], Chow-Liou-Tsai [CLT], or Andrews [A1,A2] for literature.

Let $X_{0}: S^{n} \rightarrow \mathbb{R}^{n+1}$ be a smooth parametrization of $M_{0}=X_{0}\left(S^{n}\right)$. The expansion is described by the equation

$$
\left\{\begin{array}{l}
\frac{\partial X}{\partial t}=f\left(\lambda_{1}, \lambda_{2}, \ldots . ., \lambda_{n}\right) \cdot N \\
X(0)=X_{0}
\end{array}\right.
$$

where $\lambda_{1}, \lambda_{2}, \ldots ., \lambda_{n}$ are the principal radii and $N$ is the unit outward normal to the hypersurface $M_{t}:=X_{t}\left(S^{n}\right)$. Here we assume $f \in C^{\infty}\left(\Gamma^{+}\right)$is a positive symmetric real-valued function defined on the positive cone

$$
\Gamma^{+}=\left\{\left(x_{1}, x_{2}, \ldots, x_{n}\right) \in \mathbb{R}^{n}: x_{i}>0,1 \leq i \leq n\right\}
$$

satisfying the strict parabolicity condition $\frac{\partial f}{\partial \lambda_{i}}>0$ on $\Gamma^{+}$for all $1 \leq i \leq n$. Because $f$ is symmetric, the order of $\lambda_{1}, \lambda_{2}, \ldots, \lambda_{n}$ appearing in $f\left(\lambda_{1}, \lambda_{2}, \ldots, \lambda_{n}\right)$ is irrelevant. Under certain additional assumptions on the curvature function $f$ analogous to those considered by Urbas [U1], et al, we prove that the evolving hypersurfaces remain smooth, strictly convex and expand to infinity while their shapes become round asymptotically. In particular, after an appropriate rescaling, the support functions of $M_{t}$ converge to the constant 1 in $C^{1}$-norm.

The main observation of this paper is that we may remove the homogeneity assumption on $f$ as imposed on the case of homogeneous expanding flows and still get results similar to (but weaker) the homogeneous case. However, the concavity assumption on $f$ as required in the homogeneous expanding flows is still needed in order to go from $C^{2}\left(S^{n} \times[0, T)\right)$ to $C^{2, \alpha}\left(S^{n} \times[0, T)\right)$ estimate, after that we can quickly obtain all the higher derivatives estimates by parabolic bootstraps argument and hence establish the long time existence of the solution. We believe such concavity assumption is crucial here due to the lack of powerful a priori estimates for rather general nonlinear parabolic differential equations.

\footnotetext{
*Received August 30, 1997; accepted for publication February 5, 1998.

${ }^{\dagger}$ School of Mathematics, University of Minnesota, Minneapolis, MN 55455, USA (bchow@ math.umn.edu).

$\ddagger$ Department of Mathematics, Chung Cheng University, Chiayi 621, TAIWAN, (dhtsai@math. ccu.edu.tw).
} 
Owing to the gradient estimate by Chow and Gulliver [CG] (see Proposition 3.3 below), for hypersurface expansion, as long as we have the long time existence, the asymptotic shape of the evolving hypersurface must be round. This phenomenon is quite different from the contraction flows. Recently, Andrews [A1] has shown that for certain type of contraction flows it is possible to have ellipsoid as limiting shape.

2. Main result. Let $\left(S^{n}, g\right)$ denote the unit $n$-sphere with standard metric $g_{i j}$, $\nabla$ the covariant derivative acting on tensors, and $S_{+}^{2} T^{*} S^{n}$ the bundle of symmetric covariant positive 2-tensors on $S^{n}$. Let $F: S_{+}^{2} T^{*} S^{n} \rightarrow \mathbb{R}^{+}$be the smooth function such that $F(\alpha)$ depends only on the eigenvalues of $\alpha \in S_{+}^{2} T^{*} S^{n}$ with respect to $g$ and satisfies

$$
F(\alpha)=f\left(\alpha_{1}, \alpha_{2}, \ldots ., \alpha_{n}\right)
$$

where $\alpha_{1}, \alpha_{2}, \ldots . ., \alpha_{n}$ are the positive eigenvalues of $\alpha$ and $f$ is the curvature function given in equation (1.1). Also let $u(x, t)$ be the support function of the convex hypersurface $M_{t}$. (We shall show that the convexity is preserved.) It is well known (see Tso [TS] or Urbas [U1]) that the hypersurface expansion, in terms of its support function $u(x, t)$, is equivalent to the following single parabolic evolution equation on $S^{n}$

$$
\left\{\begin{array}{l}
\partial_{t} u(x, t)=F(\nabla \nabla u+u \cdot g), \quad(x, t) \in S^{n} \times[0, T) \\
u(x, 0)=u_{0}(x)
\end{array}\right.
$$

where $u_{0}(x)$ is the support function of the initial uniformly convex hypersurface $M_{0}$ and the eigenvalues of the symmetric 2-tensor $\nabla_{i} \nabla_{j} u+u \cdot g_{i j}$ are the principal radii of the convex hypersurface $M_{t}$.

The following main assumptions on $f$ are imposed throughout this paper:

$$
\left\{\begin{array}{l}
f\left(\lambda_{1}, \lambda_{2}, \ldots, \lambda_{n}\right) \in C^{\infty}\left(\Gamma^{+}\right) \cap C^{0}\left(\bar{\Gamma}^{+}\right), \\
f \text { is a concave positive symmetric real-valued function on } \Gamma^{+}, \\
\frac{\partial f}{\partial \lambda_{i}}\left(\lambda_{1}, \lambda_{2}, \ldots, \lambda_{n}\right)>0 \text { on } \Gamma^{+} \text {for all } 1 \leq i \leq n .
\end{array}\right.
$$

In this paper we shall establish the following result:

THEOREM 2.1. Assume $f$ satisfies (2.4) and either one of the following two conditions $^{1}$ similar to Urbas [U1]:

$$
\text { (i). } f=0 \text { on the boundry } \partial \Gamma^{+} \text {of } \Gamma^{+},
$$

$$
\text { (ii). the function } g\left(\lambda_{1}, \lambda_{2}, \ldots, \lambda_{n}\right):=\frac{1}{f\left(\frac{1}{\lambda_{1}}, \frac{1}{\lambda_{2}}, \ldots, \frac{1}{\lambda n}\right)} \text { is concave on } \Gamma^{+} \text {. }
$$

Then the hypersurfaces $M_{t}$ remain smooth, strictly convex and there exists a unique solution $u(x, t) \in C^{\infty}\left(S^{n} \times[0, \infty)\right)$ to equation (2.3) satisfying

$$
\nabla \nabla u+u \cdot g>0 \quad \text { on } \quad S^{n} \times[0, \infty)
$$

such that $\lim _{t \rightarrow \infty} u_{\min }(t)=\infty$, where $u_{\min }(t)=\min _{x \in S^{n}} u(x, t)$. Moreover there exists a constant $C$ depending only on the initial condition $u_{0}(x)$ such that

$$
|\nabla u(x, t)| \leq C \quad \text { for all } \quad(x, t) \in S^{n} \times[0, \infty) .
$$

\footnotetext{
${ }^{1}$ We use either one of these two conditions to obtain the lower bound of $h_{i j}$.
} 
As a consequence, there exists a solution to the o.d.e.

$$
\frac{d R}{d t}=F\left(R \cdot g_{i j}\right)=f(R, R, \ldots, R)
$$

on $[0, \infty)$ such that

$$
u_{\min }(t) \leq R(t) \leq u_{\max }(t)
$$

and the support function $\tilde{u}=\frac{u}{R}$ of the rescaled hypersurface $\tilde{M}=\frac{M}{R}$ satisfies

$$
\|\tilde{u}(\cdot, t)-1\|_{C^{1}\left(S^{n}\right)} \leq \frac{C}{R(t)}
$$

for all $t \in[0, \infty)$. Furthermore the behavior of the expansion is improving in the following sense:

(a). $\frac{u_{\max }(t)}{R(t)}$ is dereasing, (b). $\frac{u_{\min }(t)}{R(t)}$ is increasing, (c). $\frac{u_{\max }(t)}{u_{\min }(t)}$ is decreasing on $[0, \infty)$.

The geometric meaning of Theorem 2.1 is that there exists a unique one-parameter family of smooth, strictly convex hypersurfaces satisfying equation (1.1) which expand to infinity. Moreover, the shapes of the hypersurfaces become round asymptotically in the sense that if one rescales the solution appropriately, the support functions of the rescaled hypersurfaces converge uniformly to the constant 1 in $C^{1}$-norm.

Before proceeding to the proof of the theorem, we provide some examples which satisfy the hypotheses of the theorem. For any integer $m$ with $1 \leq m \leq n$, introduce the $m$-th elementary symmetric function $S_{m}$ as

$$
S_{m}\left(\lambda_{1}, \lambda_{2}, \ldots ., \lambda_{n}\right)=S_{m}(\lambda)=\sum_{1 \leq i_{1}<\cdots<i_{m} \leq n} \lambda_{i_{1}} \cdots \lambda_{i_{m}},
$$

where $\lambda=\left(\lambda_{1}, \lambda_{2}, \ldots ., \lambda_{n}\right)$. For convenience, we let $S_{0}=1$. For any integers $1 \leq p \leq$ $m \leq n$, there exists the well-known inequality

$$
\left(\frac{S_{m}(a+b)}{S_{m-p}(a+b)}\right)^{1 / p} \geq\left(\frac{S_{m}(a)}{S_{m-p}(a)}\right)^{1 / p}+\left(\frac{S_{m}(b)}{S_{m-p}(b)}\right)^{1 / p}
$$

for any vectors $a=\left(a_{1}, a_{2}, \ldots, a_{n}\right), b=\left(b_{1}, b_{2}, \ldots ., b_{n}\right)$ with $a_{i}, b_{i}>0$ for all $i$, where the equality holds if and only if $m=p=1$ or $a_{i}=c \cdot b_{i}$ for some positive constant $c$ for all $i$. This inequality would clearly imply the concavity of the function $\left(\frac{S_{m}(\lambda)}{S_{m-p}(\lambda)}\right)^{1 / p}$ on the positive cone $\Gamma^{+}$for any $1 \leq p \leq m \leq n$. Interesting special cases are

$$
\left(\frac{S_{m}(\lambda)}{S_{m-m}(\lambda)}\right)^{1 / m}=S_{m}\left(\lambda_{1}, \lambda_{2}, \ldots, \lambda_{n}\right)^{1 / m}
$$

and

$$
\left(\frac{S_{n}(\lambda)}{S_{n-m}(\lambda)}\right)^{1 / m}=S_{m}\left(\frac{1}{\lambda_{1}}, \frac{1}{\lambda_{2}}, \ldots, \frac{1}{\lambda_{n}}\right)^{-1 / m}
$$


for any $1 \leq m \leq n$. For a function $f$ on $\Gamma^{+}$of the form

$$
f=F\left(S_{m}\left(\lambda_{1}, \lambda_{2}, \ldots, \lambda_{n}\right)^{1 / m}\right)
$$

or

$$
f=F\left(S_{m}\left(\frac{1}{\lambda_{1}}, \frac{1}{\lambda_{2}}, \ldots, \frac{1}{\lambda_{n}}\right)^{-1 / m}\right),
$$

where $F(\rho):(0, \infty) \rightarrow(0, \infty)$ is an arbitrary smooth increasing function, the if and only if condition for $f$ to be concave $\Gamma^{+}$is that $F^{\prime \prime} \leq 0$ everywhere. Since if $F^{\prime \prime}>0$ somewhere on an interval $(a, b), f$ would not be concave along some segment of the ray $\vec{r}=\{(t, t, \ldots, t): t>0\}$.

In the following examples, we understand that $\lambda_{1}, \lambda_{2}, \ldots ., \lambda_{n}$ denote the principal radii of the convex hypersurface and $K$ and $H$ represent the Gauss and mean curvatures respectively.

EXAMPLE 1. Take

$$
f=F\left(S_{m}\left(\frac{1}{\lambda_{1}}, \frac{1}{\lambda_{2}}, \ldots ., \frac{1}{\lambda_{n}}\right)^{-1 / m}\right),
$$

where $F(\rho):(0, \infty) \rightarrow(0, \infty)$ is an arbitrary smooth increasing function, continuous on $[0, \infty)$, with $F(0)=0$ and $F^{\prime \prime} \leq 0$ everywhere. Then $f$ satisfies (2.4) and (2.5). Special cases are

$$
f=F\left(\frac{1}{H}\right) \quad(m=1)
$$

and

$$
f=F\left(\frac{1}{K^{1 / n}}\right) \quad(m=n) .
$$

In the second case, the assumption $F(0)=0$ can be removed. See Chow-Tsai [CT2].

EXAMPLE 2. For any smooth increasing function $F(\rho):(0, \infty) \rightarrow(0, \infty)$, continuous on $[0, \infty)$, satisfying

$$
F^{\prime \prime}(\rho) \leq 0
$$

and

$$
F^{\prime \prime}(\rho) \cdot \rho+2 F^{\prime}(\rho)-2 \frac{F^{\prime}(\rho)^{2} \cdot \rho}{F(\rho)} \geq 0
$$

everywhere on $\rho \in(0, \infty)^{2}$, the function

$$
f=F\left(S_{m}\left(\lambda_{1}, \lambda_{2}, \ldots, \lambda_{n}\right)^{1 / m}\right)
$$

or

$$
f=F\left(S_{m}\left(\frac{1}{\lambda_{1}}, \frac{1}{\lambda_{2}}, \ldots ., \frac{1}{\lambda_{n}}\right)^{-1 / m}\right)
$$

will satisfy both assumptions (2.4) and (2.6). ${ }^{3}$

Some possible choices of $F$ for the above two examples are:

\footnotetext{
${ }^{2}$ The condition (2.10) is equivalent to the concavity of $\frac{1}{F\left(\frac{1}{z}\right)}$ on $z \in(0, \infty)$.
}

${ }^{3}$ The borderline case of $(2.10)$ is the o.d.e.

$$
F^{\prime \prime}(\rho) \cdot \rho+2 F^{\prime}(\rho)-2 \frac{F^{\prime}(\rho)^{2} \cdot \rho}{F(\rho)}=0 .
$$


1. $F(\rho)=\rho^{\alpha}, \alpha \in(0,1]$,

2. $F(\rho)=\frac{c_{2} \cdot \rho}{c_{1} \cdot \rho+1}$, where $c_{1} \geq 0, c_{2}>0$ are any constants,

3. $F(\rho)=\log (\rho+c)$ for some suitable constant $c>0$.

3. Proof of Theorem 2.1. Let $h_{i j}=\nabla_{i} \nabla_{j} u+u \cdot g_{i j}$ be the components of the 2-tensor $\nabla \nabla u+u \cdot g$ with respect to some smooth local orthonormal frame field on $S^{n}$. Standard theory for strictly parabolic equations implies the existence of a unique smooth solution $u(x, t)$ to $(2.3)$ on $S^{n} \times[0, T)$ for some short time $T>0$ and we have $h_{i j}>0$ on the short time $[0, T)$. We shall prove its long-time existence based on a priori estimates on solution of (2.3).

Define the linearization of $F$ at the point $h=h_{i j}$ as

$$
D F_{h}(\alpha):=\left.\frac{d}{d s}\right|_{s=0} F(h+s \cdot \alpha) \text { for all } \alpha \in S^{2} T^{*} S^{n}
$$

where $S^{2} T^{*} S^{n}$ is the bundle of symmetric covariant 2-tensors on $S^{n}$. For simplicity, we also use the summation notation $F_{i j}^{\prime}(h) \alpha_{i j}$ to denote $D F_{h}(\alpha)$. Similarly, we define

$$
F_{k l ; m n}^{\prime \prime}(h) \alpha_{k l} \beta_{m n}:=\left.\left.\frac{d}{d t}\right|_{t=0} \frac{d}{d s}\right|_{s=0} F(h+s \cdot \alpha+t \cdot \beta) \quad \text { for all } \alpha, \beta \in S^{2} T^{*} S^{n} .
$$

Denote the eigenvalues of the tensor $h_{i j}$ by $\lambda_{1}, \lambda_{2}, \ldots . ., \lambda_{n}$, which are the principal radii of the hypersurfaces. It is easy to see

$$
\begin{aligned}
& F_{i j}^{\prime}(h) g_{i j}=\operatorname{trace} F^{\prime}=\frac{\partial f}{\partial \lambda_{1}}+\ldots .+\frac{\partial f}{\partial \lambda_{n}} \\
& F_{i j}^{\prime}(h) h_{i j}=\lambda_{1} \frac{\partial f}{\partial \lambda_{1}}+\ldots .+\lambda_{n} \frac{\partial f}{\partial \lambda_{n}} .
\end{aligned}
$$

Also we note that the tensor $h_{i j}$ satisfies the identity $\nabla_{k} h_{i j}=\nabla_{i} h_{k j}$, which is analogous to the Codazzi equation for the second fundamental form. In fact, for any smooth function $v$ on $S^{n}$, the symmetric 2-tensor $t_{i j}=\nabla_{i} \nabla_{j} v+v \cdot g_{i j}$ on $S^{n}$ satisfies the identity

$$
\nabla_{k} t_{i j}=\nabla_{i} t_{k j}
$$

The proof is based on the formula for commuting covariant derivatives, i.e.,

$$
\nabla_{k} \nabla_{i} \nabla_{j} v-\nabla_{i} \nabla_{k} \nabla_{j} v=-R_{k i j l} \nabla_{l} v
$$

where the Riemann curvature tensor $R_{k i j l}$ on $S^{n}$ is given by

$$
R_{k i j l}=g_{k l} g_{i j}-g_{k j} g_{i l} \text {. }
$$

We shall need the following two simple inequalities which depend only on the concavity of $f$. They will be used to obtain the upper bound of $h_{i j}$.

We can converted it into a Bernoulli equation using the new variable $y=\frac{F^{\prime}(\rho)}{F(\rho)}$ and solve it to get the general solution of $(2.11)$, which is

$$
F(\rho)=\frac{c_{2} \cdot \rho}{c_{1} \cdot \rho+1}
$$

where $c_{1} \geq 0, c_{2}>0$ are any constants. 
Lemma 3.2. (1). Let $\lambda=\left(\lambda_{1}, \lambda_{2}, \ldots . ., \lambda_{n}\right)$. We have

$$
\lambda_{1} \frac{\partial f}{\partial \lambda_{1}}(\lambda)+\ldots .+\lambda_{n} \frac{\partial f}{\partial \lambda_{n}}(\lambda) \leq n f(\lambda)
$$

for all $\lambda \in \Gamma^{+}$.

(2). There exist positive constants $A, B$ depending on $f$ such that

$$
f(\lambda) \leq A+B\left(\lambda_{1}+\lambda_{2}+\cdots+\lambda_{n}\right)
$$

for all $\lambda \in \Gamma^{+}$.

Proof. To prove (3.12), consider

$$
\begin{aligned}
f\left(\lambda_{1}, \lambda_{2}, \ldots ., \lambda_{n}\right) & \geq f\left(\lambda_{1}, \lambda_{2}, \ldots ., \lambda_{n}\right)-f\left(0, \lambda_{2}, \ldots ., \lambda_{n}\right) \\
& =\lambda_{1} \frac{\partial f}{\partial \lambda_{1}}\left(\lambda_{1}^{*}, \lambda_{2}, \ldots ., \lambda_{n}\right) \geq \lambda_{1} \frac{\partial f}{\partial \lambda_{1}}\left(\lambda_{1}, \lambda_{2}, \ldots ., \lambda_{n}\right)
\end{aligned}
$$

where $0<\lambda_{1}^{*}<\lambda_{1}$. Similarly we have

$$
f\left(\lambda_{1}, \lambda_{2}, \ldots ., \lambda_{n}\right) \geq \lambda_{i} \frac{\partial f}{\partial \lambda_{i}}\left(\lambda_{1}, \lambda_{2}, \ldots ., \lambda_{n}\right),
$$

for all $i=2,3, \ldots, n$. For $(3.13)$, since $f$ is concave on $\Gamma^{+}$, we have

$$
f(\lambda+\vec{\mu}) \leq f(\lambda)+\mu\left[\frac{\partial f}{\partial \lambda_{1}}(\lambda)+\ldots .+\frac{\partial f}{\partial \lambda_{n}}(\lambda)\right]
$$

for all $\lambda \in \Gamma^{+}, \vec{\mu}=(\mu, \mu, \ldots, \mu), \mu>0$. Let $C$ be the compact set

$$
C=\left\{\lambda \in \bar{\Gamma}^{+}:\left|\lambda_{i}\right| \leq 1 \text { for all } i\right\} .
$$

If $\lambda \in \Gamma^{+}$and $\lambda \notin C$, there exists some $i$ such that $\lambda_{i}>1$. We may assume $\lambda_{1} \leq \lambda_{2} \leq$ $\ldots \leq \lambda_{n}$ and $\lambda_{n}>1$ for $\operatorname{such} \lambda$. Now

$$
\begin{aligned}
f(\lambda) & \leq f\left(\lambda_{n}, \lambda_{n}, \ldots, \lambda_{n}\right) \\
& \leq f(\overrightarrow{1})+\left(\lambda_{n}-1\right)\left[\frac{\partial f}{\partial \lambda_{1}}(\overrightarrow{1})+\ldots .+\frac{\partial f}{\partial \lambda_{n}}(\overrightarrow{1})\right] \\
& \leq f(\overrightarrow{1})+\left[\frac{\partial f}{\partial \lambda_{1}}(\overrightarrow{1})+\ldots .+\frac{\partial f}{\partial \lambda_{n}}(\overrightarrow{1})\right] \cdot\left(\lambda_{1}+\lambda_{2}+\cdots+\lambda_{n}\right),
\end{aligned}
$$

where $\overrightarrow{1}=(1,1, \ldots, 1)$. Choose $A=\max _{C} f$ and $B=\frac{\partial f}{\partial \lambda_{1}}(\overrightarrow{1})+\ldots+\frac{\partial f}{\partial \lambda_{n}}(\overrightarrow{1})$.

3.1. The gradient estimate. Without loss of generality, we may assume the initial hypersurface $M_{0}$ enclose the origin of $\mathbb{R}^{n+1}$. Therefore $u_{0}(x) \geq \delta>0$ for some constant $\delta>0$ and $u(x, t)$ will remain positive during the evolution. We will see later that the quantitative behavior of $u(x, t)$ is close to a solution $R(t)$ to the o.d.e. $\frac{d R}{d t}=F\left(R \cdot g_{i j}\right)=f(R, R, \ldots . ., R), R(0) \in\left(u_{\min }(0), u_{\max }(0)\right)$. Because $u(\cdot, t)>0$ is the support function of the strictly convex hypersurface $M_{t}$ (see Corollary 3.8), it is not difficult to see that

$$
|\nabla u(p, t)| \leq u\left(\frac{\nabla u(p, t)}{|\nabla u(p, t)|}, t\right)
$$


for all $(p, t) \in S^{n} \times[0, T)$ whenever $|\nabla u(p, t)| \neq 0^{4}$. Consequently, we obtain

$$
\sup _{S^{n}}|\nabla u(\cdot, t)| \leq \sup _{S^{n}} u(\cdot, t) \quad \text { for all } t \in[0, T) .
$$

This rough estimate can be refined substantially. In fact, there is a uniform bound for the gradient of $u$. This is a special case of Theorem 3.1.(iv) in Chow-Gulliver [CG], which provides a uniform gradient estimate for more general equations based on an Aleksandrov reflection argument. To get a feeling of the geometric aspects of Aleksandrov reflection and its application to conformal flows on $S^{n}$, the readers can see the recent paper by Chow [C]. The implications of the gradient estimate are twofold: first, it implies the asymptotic roundness of the hypersurfaces as long as we have the long time existence of the evolution; second, it tells us how to rescale the solution in a precise way. The statement is

Proposition 3.3. There exists a positive constant $C$ depending only on the initial condition $u_{0}$ such that, as long as the solution to (2.3) exists and $h_{i j}>0$ on $S^{n} \times[0, T)$, the support function $u(x, t)$ satisfies the uniform gradient estimate

$$
|\nabla u(x, t)| \leq C
$$

for all $(x, t) \in S^{n} \times[0, T)$. In particular, since the diameter of $S^{n}$ is $\pi$, we have

$$
u_{\max }(t)-u_{\min }(t) \leq C \cdot \pi
$$

on $[0, T)$.

REMARK 1. We only need the parabolic condition $\frac{\partial f}{\partial \lambda_{i}}>0$ to get the gradient estimate (3.15). The assumptions in (2.5) and (2.6) and that $f$ being concave and positive on $\Gamma^{+}$are not necessary. However, they are required for the higher derivatives estimates.

It is worth pointing out that our equation (2.3) is geometrical and therefore any estimate related to it may depend on the geometry of the setting. For example if, in the 1-dimensional case, we replace the initial imbedded closed convex curve $M_{0}$ in $\mathbb{R}^{2}$ by an immersed closed convex curve with loops, then the gradient estimate (3.15) is no longer valid. See Tsai [T]. A geometric explanation of this phenomenon can be given easily as follows. Imagine a cardioidlike curve with one little loop $L_{2}$ and call the rest $L_{1}$. Suppose we also position our coordinate system so that the origin $O$ is enclosed by the little loop $L_{2}$. The support function $u_{0}(x)$ is now a periodic function with domain $[0,4 \pi]$ and is everywhere positive. It has larger values on $L_{1}$, smaller values on the little loop $L_{2}$. Also, for any point $p$ on $L_{1}$, which has small curvature, the expansion speed of $p$ is much faster than the expansion speed of any point $q$ on the little loop $L_{2}$, which has large curvature. Therefore the difference $u_{\max }(t)-u_{\min }(t)$ will not be bounded above by any positive constant as time evolves.

3.2. The second derivative estimate. Regarding the second derivatives estimates of $u$, we need to compute the evolution equations of $h_{i j}$ and $h^{i j}$ first.

LEMma 3.4. (1).

$$
\begin{aligned}
\partial_{t} h_{i j}= & F_{k l}^{\prime} \nabla_{k} \nabla_{l} h_{i j}+F_{k l ; m n}^{\prime \prime} \nabla_{i} h_{k l} \nabla_{j} h_{m n}+\left(F+F_{k l}^{\prime} h_{k l}\right) g_{i j}-\left(t r F^{\prime}\right) h_{i j} \\
& +F_{i k}^{\prime} h_{j k}-F_{j k}^{\prime} h_{i k}
\end{aligned}
$$

\footnotetext{
${ }^{4}$ This is obtained by computing $\left.\frac{d}{d s}\right|_{s=0} u(\alpha(s), t)$ along a curve $\alpha(s) \in S^{n}$ with $\alpha(0)=p, \dot{\alpha}(0)=$ $\frac{\nabla u(p, t)}{|\nabla u(p, t)|}$ and use the definition of support function.
} 
(2).

$$
\begin{aligned}
\partial_{t} h^{i j}= & F_{k l}^{\prime} \nabla_{k} \nabla_{l} h^{i j}-\left(F+F_{k l}^{\prime} h_{k l}\right) h^{i p} h^{j p}+\left(t r F^{\prime}\right) h^{i j} \\
& -h^{i r} h^{j s}\left[2 F_{k m}^{\prime} h^{n l}+F_{k l ; m n}^{\prime \prime}\right] \nabla_{r} h_{k l} \nabla_{s} h_{m n} \\
& -h^{i p} h^{j q} \cdot\left(F_{p k}^{\prime} h_{q k}-F_{q k}^{\prime} h_{p k}\right)
\end{aligned}
$$

where $F, F^{\prime}$, and $F^{\prime \prime}$ are all evaluated at $h$ and $\operatorname{tr} F^{\prime}=F_{k l}^{\prime}(h) g_{k l}$.

Proof. The computations are very similar to those as in Urbas [U1], they are therefore omitted.

3.2.1. The upper bound of $h_{i j}$. We only need the concavity of $f$ on $\Gamma^{+}$to get the bound on $|\nabla \nabla u|$, which in turn implies the upper bound of $h_{i j}$.

Proposition 3.5. If $u \leq M$ on $S^{n} \times[0, T)$ for some positive constant $M$, then

$$
|\nabla \nabla u(x, t)| \leq \tilde{C} \quad \text { for all } \quad(x, t) \in S^{n} \times[0, T),
$$

where $\tilde{C}<\infty$ is a constant depending on $M, F, u_{0}$ and $T$.

Proof. We shall let $C$ denote any constant depending only on $F, u_{0}$ and $T$, where $C$ may change from line to line. Let

$$
\tilde{H}=g^{i j} h_{i j}=\Delta u+n u .
$$

By (3.16), we find

$$
\partial_{t} \tilde{H}=F_{k l}^{\prime} \nabla_{k} \nabla_{l} \tilde{H}+n\left(F+F_{k l}^{\prime} h_{k l}\right)-\left(t r F^{\prime}\right) \tilde{H}+F_{k l ; m n}^{\prime \prime} \nabla_{i} h_{k l} \nabla_{i} h_{m n} .
$$

The concavity of $f$ would then imply (see Caffarelli-Nirenberg-Spruck [CNS])

$$
F_{k l ; m n}^{\prime \prime} \nabla_{i} h_{k l} \nabla_{i} h_{m n} \leq 0
$$

Therefore

$$
\partial_{t} \tilde{H} \leq F_{k l}^{\prime} \nabla_{k} \nabla_{l} \tilde{H}+n\left(F+F_{k l}^{\prime} h_{k l}\right)
$$

since $_{(}\left(t r F^{\prime}\right) \tilde{H} \geq 0$. Applying the inequalities (3.12) and (3.13) to above yields

$$
\partial_{t} \tilde{H} \leq F_{k l}^{\prime} \nabla_{k} \nabla_{l} \tilde{H}+C \cdot \tilde{H}+C .
$$

We thus find

$$
0<\tilde{H} \leq C \quad \text { on } \quad S^{n} \times[0, T)
$$

by the maximum principle. Observing that

$$
0<\lambda_{i}<\lambda_{1}+\cdots+\lambda_{n}=\tilde{H} \leq C
$$

for any $1 \leq i \leq n$ and

$$
|\nabla \nabla u(x, t)|^{2}=\left(\lambda_{1}-u\right)^{2}+\left(\lambda_{2}-u\right)^{2}+\cdots\left(\lambda_{n}-u\right)^{2} \leq \tilde{C},
$$

the proof of the proposition is done. finite.

Since $h_{i j}=\nabla_{i} \nabla_{j} u+u g_{i j}$, the upper bound of it is established as long as $u$ stays 
3.2.2. The lower bound of $h_{i j}$ and the preserving of convexity. We want to show that the convexity of the evolving hypersurfaces is preserved if we impose either assumption (2.5) or (2.6) on $f$. As a consequence, all of the principal radii $\lambda_{1}, \lambda_{2}, \ldots, \lambda_{n}$ will have positive lower and upper bounds as long as $u$ is bounded. First we have

LEMMA 3.6. For all $t \in[0, T)$, we have

$$
\min _{S^{n}} F(\nabla \nabla u(\cdot, t)+u(\cdot, t) \cdot g) \geq \min _{S^{n}} F(\nabla \nabla u(\cdot, 0)+u(\cdot, 0) \cdot g)>0
$$

Proof. Let $w(x, t)=\partial_{t} u=F(\nabla \nabla u(x, t)+u(x, t) \cdot g)$. Compute

$$
\begin{aligned}
\partial_{t} w & =F_{i j}^{\prime}(h) \cdot\left[\nabla_{i} \nabla_{j} w+w \cdot g_{i j}\right] \\
& =F_{i j}^{\prime}(h) \nabla_{i} \nabla_{j} w+F_{i j}^{\prime}(h) g_{i j} \cdot w .
\end{aligned}
$$

Since

$$
F_{i j}^{\prime}(h) g_{i j}=\frac{\partial f}{\partial \lambda_{1}}+\ldots .+\frac{\partial f}{\partial \lambda_{n}}>0
$$

on $S^{n} \times[0, T)$, we can apply the weak maximum principle to conclude

$$
w(x, t)=f\left(\lambda_{1}(x, t), \ldots . ., \lambda_{n}(x, t)\right) \geq \min _{S^{n}} w(x, 0) \geq f(\epsilon, \epsilon, \ldots ., \epsilon)>0
$$

for some $\epsilon>0$ and for all $(x, t) \in S^{n} \times[0, T)$. (3.21) is proved.

Proposition 3.7. If $f$ satisfies either assumption (2.5) or (2.6), there exist a positive constant $C_{1}$ depending on $F, u_{0}$ and $T$ such that

$$
h_{i j} \geq C_{1} \cdot g_{i j} \quad \text { on } \quad S^{n} \times[0, T) .
$$

Proof. Case 1. Assume $f$ satisfies assumption (2.5).

Since we impose the barrier condition $f=0$ on $\partial \Gamma^{+}$, it will force each $\lambda_{i}$ to stay strictly away from 0 for all $t \in[0, T)$ due to (3.22). That is, $\left(\lambda_{1}, \lambda_{2}, \ldots ., \lambda_{n}\right) \in \Omega$ on $S^{n} \times[0, T)$, where $\Omega$ is a closed subset of $\Gamma^{+}$with $\Omega \cap \partial \Gamma^{+}=\phi$. The set $\Omega$ is also bounded because of (3.20). Hence $\Omega$ is a compact set. The proof is done.

Case 2. Assume $f$ satisfies assumption (2.6).

Recall we have the equation

$$
\begin{aligned}
\partial_{t} h^{i j}= & F_{k l}^{\prime} \nabla_{k} \nabla_{l} h^{i j}-\left(F+F_{k l}^{\prime} h_{k l}\right) h^{i p} h^{j p}+\left(t r F^{\prime}\right) h^{i j} \\
& -h^{i r} h^{j s}\left[2 F_{k m}^{\prime} h^{n l}+F_{k l ; m n}^{\prime \prime}\right] \nabla_{r} h_{k l} \nabla_{s} h_{m n} \\
& -h^{i p} h^{j q} \cdot\left(F_{p k}^{\prime} h_{q k}-F_{q k}^{\prime} h_{p k}\right) .
\end{aligned}
$$

Now let us suppose that the maximum eigenvalue of $\left[h^{i j}\right]$ over $S^{n}$ at time $t$ is attained at a point $p_{t} \in S^{n}$. By a rotation of the frame, we may assume that $h^{11}$ is the maximum eigenvalue and $h^{i j}=0$ for $i \neq j$. We get

$$
\begin{aligned}
\partial_{t} h^{11}= & F_{k l}^{\prime} \nabla_{k} \nabla_{l} h^{11}-\left(F+F_{k l}^{\prime} h_{k l}\right)\left(h^{11}\right)^{2}+\left(t r F^{\prime}\right) h^{11} \\
& -\left(h^{11}\right)^{2} \cdot\left[2 F_{k m}^{\prime} h^{n l}+F_{k l ; m n}^{\prime \prime}\right] \nabla_{1} h_{k l} \nabla_{1} h_{m n}
\end{aligned}
$$


By our assumption (2.6), we have (see Urbas [U1])

$$
-\left(h^{11}\right)^{2} \cdot\left[2 F_{k m}^{\prime} h^{n l}+F_{k l ; m n}^{\prime \prime}\right] \nabla_{1} h_{k l} \nabla_{1} h_{m n} \leq 0
$$

Assuming $0<\lambda_{1} \leq \lambda_{2} \leq \cdots \leq \lambda_{n}$ at $p_{t} \in S^{n}$, we have $h^{11}=\frac{1}{\lambda_{1}}$ and

$$
\begin{aligned}
& -\left(F+F_{k l}^{\prime} h_{k l}\right)\left(h^{11}\right)^{2}+\left(t r F^{\prime}\right) h^{11} \\
= & -F\left(\frac{1}{\lambda_{1}}\right)^{2}-\left[\lambda_{1} \frac{\partial f}{\partial \lambda_{1}}+\ldots .+\lambda_{n} \frac{\partial f}{\partial \lambda_{n}}\right]\left(\frac{1}{\lambda_{1}}\right)^{2}+\left[\frac{\partial f}{\partial \lambda_{1}}+\ldots .+\frac{\partial f}{\partial \lambda_{n}}\right]\left(\frac{1}{\lambda_{1}}\right) \\
\leq & -F\left(\frac{1}{\lambda_{1}}\right)^{2}<0 .
\end{aligned}
$$

Therefore we conclude

$$
\partial_{t} h^{11} \leq 0 \quad \text { at the maximum point } p_{t} \in S^{n} .
$$

Again the maximum principle implies the upper bound of $h^{i j}$, which is the same as the lower bound of $h_{i j}$.

COROLlaRY 3.8. (preserving the convexity) If $f$ satisfies either assumption (2.5) or. (2.6) and $u \leq M$ on $S^{n} \times[0, T)$, there exist two positive constants $C_{1}$ and $C_{2}$ depending on $M, F, u_{0}$ and $T$ such that

$$
0<C_{1} \cdot g_{i j} \leq h_{i j} \leq C_{2} \cdot g_{i j} \quad \text { on } S^{n} \times[0, T) .
$$

This means that the evolving hypersurface is uniformly convex as long as $u$ is finite.

3.2.3. Long time existence. So far we have obtained the estimates on the first and second space derivatives of $u$ and the bound of the tensor $h_{i j}$, whenever $u$ stays finite. This will guarantee the uniform parabolicity of our nonlinear equation (2.3) as long as $u$ is finite. By the same argument as in Urbas [U1], we can quote the result of Krylov-Safanov [KS] to get the $C^{\alpha}$ estimate of $\frac{\partial u}{\partial t}$. Since $f$ is concave, by a result of Krylov [K] again, we also have the $C^{\alpha}$ estimate of $\nabla \nabla u$. (Both estimates are with respect to space and time.) Standard parabolic theory again allows us to derive the $C^{k, \alpha}$ estimates of $u$ provided $u$ stays finite. We hence arrive at the following conclusion.

PROPOSITION 3.9. (long time existence) Under the hypotheses of Theorem 2.1, there exists a unique smooth solution $u(x, t)$ to equation (2.3) on $S^{n} \times\left[0, T_{\infty}\right)$ such that $h_{i j}>0$ on $S^{n} \times\left[0, T_{\infty}\right)$ and $\lim _{t \rightarrow T_{\infty}} u_{\min }(t)=\infty$.

We shall see immediately that $T_{\infty}=\infty$.

3.2.4. Rescaling and convergence. By the equation $\partial_{t} u=F(\nabla \nabla u+u \cdot g)$, we can get

$$
\frac{d^{+} u_{\max }(t)}{d t} \leq F\left(u_{\max }(t) \cdot g\right) \text { and } \quad \frac{d^{-} u_{\min }(t)}{d t} \geq F\left(u_{\min }(t) \cdot g\right) .
$$

where $\frac{d^{+}}{d t}$ and $\frac{d^{-}}{d t}$ are in the Lipschitz sense. (See Hamilton [H] or Chow-Tsai [CT1].) Because $f$ is concave, we have

$$
F\left(u_{\max }(t) \cdot g\right)=f\left(u_{\max }(t), u_{\max }(t), \ldots ., u_{\max }(t)\right) \leq A+n B \cdot u_{\max }(t)
$$


by (3.13). Hence $T_{\infty}=\infty$. Moreover, based on inequalities (3.26), we can carry out exactly the same proof as in Chow-Tsai [CT1] to conclude

LEMma 3.10. (1). There exists a solution $R(t)$ to the o.d.e.

$$
\frac{d R}{d t}=F(R \cdot g)
$$

on $[0, \infty)$ such that

$$
u_{\min }(t) \leq R(t) \leq u_{\max }(t) \quad \text { for all } \quad t \in[0, \infty)
$$

(2). For any $R(t)$ satisfying (3.27) and (3.28), we have

(a). $\frac{u_{\max }(t)}{R(t)}$ is dereasing, (b). $\frac{u_{\min }(t)}{R(t)}$ is increasing, (c). $\frac{u_{\max }(t)}{u_{\min }(t)}$ is decreasing for all $t \in[0, \infty)$.

From now on we will choose one $R(t)$ satisfying (3.28) and use it to rescale the solution $u(x, t)$. Define the rescaled solution $\tilde{u}(x, t)$ as

$$
\tilde{u}(x, t)=\frac{u(x, t)}{R(t)}
$$

which is the support function of the rescaled surface $\tilde{M}=\frac{M}{R}$. Clearly we have

$$
|\tilde{u}(x, t)-1| \leq \frac{C}{R(t)}, \quad|\nabla \tilde{u}(x, t)| \leq \frac{C}{R(t)}
$$

for all $(x, t) \in S^{n} \times[0, \infty)$ and the proof of Theorem 2.1 is finished.

REMARK 2. We would like to point out that the estimate (3.15) and relation (3.28) hold as long as we have the parabolic condition $\frac{\partial f}{\partial \lambda_{i}}>0$.

4. Rescaling of solutions to some parabolic differential equations. In this last section we establish a theorem which says that under some assumptions on the parabolic partial differential equation considered, there is a natural way to rescale the solution. Similar to Lemma 3.10, the rescaling function $R(t)$ is chosen to be a solution of some ordinary differential equation with its value lying between the maximum and the minimum values of the solution $u(x, t)$ to the partial differential equation considered. Again one can see Hamilton [H] or Chow-Tsai [CT1] for the discussion and notations on the differentiability of Lipschitz functions.

Let $F\left(a_{i j}, b_{i}, x, t\right)$ be a real-valued smooth function defined on the set $\Gamma=$ $S(n) \times \mathbb{R}^{n} \times \mathbb{R} \times[0, T)$. Here $S(n)$ denotes the $n(n+1) / 2$-dimensional space of real symmetric $n \times n$ matrices. The main assumption on $F$ in this section is

$$
F(0,0, x, t) \text { is increasing in the variable } x \text { for all } t \in[0, T) .
$$

The main result in this section is

THEOREM 4.11. Let $u(x, t) \in C^{2}(M \times[0, T))$, where $T \leq \infty$, be a solution to the following parabolic differential equation

$$
\partial_{t} u=F\left(\nabla_{i} \nabla_{j} u, \nabla_{i} u, u, t\right)
$$


on any compact Riemannian manifold $(M, g)$. Here $F$ satisfies the assumption (4.30). Then there exists a solution to the o.d.e.

$$
\frac{d R}{d t}=F(0,0, R, t)
$$

on $[0, T)$ such that

$$
u_{\min }(t) \leq R(t) \leq u_{\max }(t)
$$

for all $t \in[0, T)$.

REMARK 3. The theorem also holds if we replace $(M, g)$ by a compact set $Y$ in Euclidean space with standard metric and assume that for any time $t \in[0, T)$, both $u_{\min }(t)=\min _{x \in Y} u(x, t)$ and $u_{\max }(t)=\max _{x \in Y} u(x, t)$ are attained at some interior points of $Y$, since at the interior extremum points we have $\nabla_{i} u=0$ and $\nabla_{i} \nabla_{j} u \leq 0$ or $\geq 0$.

Proof. Consider the first case when $T<\infty$. For any $t \in[0, T)$, if $u_{\max }(t)=u\left(p_{t}, t\right)$ for some $p_{t} \in M$, then

$$
\begin{aligned}
\frac{\partial u}{\partial t}\left(p_{t}, t\right) & =F\left(\nabla_{i} \nabla_{j} u, \nabla_{i} u, u, t\right) \quad \text { at } \quad\left(p_{t}, t\right) \\
& \leq F\left(0,0, u_{\max }(t), t\right)
\end{aligned}
$$

since $\nabla_{i} \nabla_{j} u$ is semi-negative at the maximum point. Similarly we have

$$
\frac{\partial u}{\partial t}\left(q_{t}, t\right) \geq F\left(0,0, u_{\min }(t), t\right)
$$

where $u_{\min }(t)=u\left(q_{t}, t\right)$ for some $q_{t} \in M$. Take an increasing sequence $T_{i} \in[0, T)$ with $\lim _{i \rightarrow \infty} T_{i}=T$ and let $R_{i}^{+}(t)$ be the solution to the following

$$
\frac{d R_{i}^{+}}{d t}=F\left(0,0, R_{i}^{+}(t), t\right), \quad R_{i}^{+}\left(T_{i}\right)=u_{\max }\left(T_{i}\right) .
$$

The domain of $R_{i}^{+}(t)$ will be at least $\left(T_{i}-\varepsilon_{i}, T_{i}\right]$ for some $\varepsilon_{i}>0$. Set

$$
f(t)=u_{\max }(t)-R_{i}^{+}(t)
$$

on $\left(T_{i}-\varepsilon_{i}, T_{i}\right]$. It will be Lipschitz on $\left(T_{i}-\varepsilon_{i}, T_{i}\right]$ and $f\left(T_{i}\right)=0$. For any $t \in$ $\left(T_{i}-\varepsilon_{i}, T_{i}\right]$, compute

$$
\begin{aligned}
& \limsup _{h \searrow 0} \frac{f(t)-f(t-h)}{h} \\
\leq & \limsup _{h \searrow 0} \frac{u_{\max }(t)-u_{\max }(t-h)}{h}-F\left(0,0, R_{i}^{+}(t), t\right)
\end{aligned}
$$

and by (4.30) and (4.34) we know

$$
\frac{d^{-} f}{d t}(t) \leq F\left(0,0, u_{\max }(t), t\right)-F\left(0,0, R_{i}^{+}(t), t\right) \leq 0
$$

whenever $f(t) \leq 0$. Therefore

$$
f(t) \geq 0 \quad \text { for all } \quad t \in\left(T_{i}-\varepsilon_{i}, T_{i}\right] .
$$


Similarly we consider $g(t)=u_{\min }(t)-R_{i}^{+}(t)$ and compute

$$
\begin{aligned}
& \liminf _{h \searrow 0} \frac{g(t)-g(t-h)}{h} \\
\geq & \liminf _{h \searrow 0} \frac{u_{\min }(t)-u_{\min }(t-h)}{h}-F\left(0,0, R_{i}^{+}(t), t\right) .
\end{aligned}
$$

Again by (4.30) and (4.35) we know

$$
\frac{d^{-} g}{d t}(t) \geq F\left(0,0, u_{\min }(t), t\right)-F\left(0,0, R_{i}^{+}(t), t\right) \geq 0,
$$

whenever $g(t) \geq 0$. Hence

$$
g(t) \leq 0 \quad \text { for all } \quad t \in\left(T_{i}-\varepsilon_{i}, T_{i}\right] .
$$

Thus we arrive at the following

$$
u_{\min }(t) \leq R_{i}^{+}(t) \leq u_{\max }(t) \quad \text { on } \quad\left(T_{i}-\varepsilon_{i}, T_{i}\right] .
$$

From (4.40) and the basic theory of o.d.e., we deduce that the domain of $R_{i}^{+}(t)$ will be at least $\left[0, T_{i}\right]$ and for each $i$ we have

$$
u_{\min }(t) \leq R_{i}^{+}(t) \leq u_{\max }(t) \quad \text { on } \quad\left[0, T_{i}\right]
$$

Consider the sequence $\left\{R_{i}^{+}\right\}_{i=1}^{\infty}$, on any compact subinterval $[0, T-\delta]$ of $[0, T)$, the domain of $R_{i}^{+}$will cover $[0, T-\delta]$ for $i$ large enough. We may assume all $R_{i}^{+}$are defined on $[0, T-\delta]$. Observe that each $R_{i}^{+}(t)$ is smooth on $[0, T-\delta]$ and if $j>i$, we have $T-\delta<T_{i}<T_{j}$ and

$$
R_{j}^{+}(t) \leq u_{\max }(t) \quad \text { on } \quad\left[T_{i}, T_{j}\right]
$$

In particular, we see

$$
R_{j}^{+}\left(T_{i}\right) \leq R_{i}^{+}\left(T_{i}\right)
$$

Therefore by the basic theory of o.d.e. again we get the monotone relation

$$
u_{\min }(t) \leq R_{j}^{+}(t) \leq R_{i}^{+}(t) \leq u_{\max }(t) \quad \text { on } \quad[0, T-\delta]
$$

for any $j>i$. Define $R^{+}(t)=\lim _{i \rightarrow \infty} R_{i}^{+}(t), t \in[0, T-\delta]$. Clearly we have

$$
u_{\min }(t) \leq R^{+}(t) \leq u_{\max }(t) \quad \text { on } \quad[0, T-\delta] .
$$

Claim: $R_{i}^{+}(t)$ converges uniformly to $R^{+}(t)$ on the interval $[0, T-\delta]$.

Proof of the claim:

For any $t \in[0, T-\delta]$ and $j>i$, we have

$$
\begin{aligned}
{\left[R_{i}^{+}(t)-R_{j}^{+}(t)\right]=} & {\left[R_{i}^{+}(0)-R_{j}^{+}(0)\right] } \\
& +\int_{0}^{t}\left[F\left(0,0, R_{i}^{+}(s), s\right)-F\left(0,0, R_{j}^{+}(s), s\right)\right] \cdot d s
\end{aligned}
$$


and hence

(4.44) $0 \leq\left[R_{i}^{+}(t)-R_{j}^{+}(t)\right] \leq\left[R_{i}^{+}(0)-R_{j}^{+}(0)\right]+\int_{0}^{t} M_{\delta} \cdot\left[R_{i}^{+}(s)-R_{j}^{+}(s)\right] \cdot d s$.

for some constant $M_{\delta}>0$ since $F$ is Lipschitz when restricted to compact sets. Gronwall's inequality implies

$$
0 \leq\left[R_{i}^{+}(t)-R_{j}^{+}(t)\right] \leq\left[R_{i}^{+}(0)-R_{j}^{+}(0)\right] \cdot e^{M_{\delta} \cdot t}
$$

for all $t \in[0, T-\delta]$. The claim is proved.

The claim would also imply that we have $F\left(0,0, R_{i}^{+}(t), t\right)$ converges uniformly to $F\left(0,0, R^{+}(t), t\right)$ on $[0, T-\delta]$ due to the mean value theorem. Therefore we secure the continuity and differentiability of $R^{+}(t)$ on the interval $[0, T-\delta]$ and moreover

$$
\frac{d R^{+}}{d t}=F\left(0,0, R^{+}(t), t\right) \quad \text { on } \quad[0, T-\delta] .
$$

Letting $\delta \rightarrow 0$, the conclusion follows. The case when $T=\infty$ is also clear. The proof of Theorem 4.11 is complete.

REMARK 4. The fact that we can squeeze a solution $R(t)$ to the o.d.e. (4.32) between $u_{\min }(t)$ and $u_{\max }(t)$ has more significance when we have the gradient estimate

$$
|\nabla u| \leq C
$$

of the solution $u$ to equation (4.31), where $C$ is a constant depending only on the initial condition $u_{0}(x)$ and when the solution $u$ will blow up as $t \rightarrow T$. In this case we have

$$
0 \leq u_{\max }(t)-u_{\min }(t) \leq C \cdot d,
$$

where $d$ is the diameter of $M$ and $\lim _{t \rightarrow T} u_{\min }(t)=\infty$. So the asymptotic behavior of $u$ is best described by $R(t)$.

Another interesting consequence is:

COROLlary 4.12. Under the hypotheses of Theorem 4.11, if in addition $F$ also satisfies all of the following

(i).. $F(0,0, x, t)$ is concave in the variable $x \in \mathbb{R}$, for all $t \in[0, T)$,

(ii). $F(0,0,0, t) \geq 0$, for all $t \in[0, T)$,

(iii). $0<u_{\min }(t)$, for all $t \in[0, T)$

then for any $R(t)$ satisfying (4.32) and (4.33) we have

(a). $\frac{u_{\max }(t)}{R(t)}$ is dereasing, (b). $\frac{u_{\min }(t)}{R(t)}$ is increasing, (c). $\frac{u_{\max }(t)}{u_{\min }(t)}$ is decreasing on $[0, T)$.

Proof. To prove (a), note that $u_{\max }(t) / R(t)$ is Lipschitz on $[0, T)$ and get

$$
\frac{d^{+}}{d t}\left(\frac{u_{\max }(t)}{R(t)}\right) \leq \frac{R(t) F\left(0,0, u_{\max }(t), t\right)-u_{\max }(t) F(0,0, R(t), t)}{R^{2}(t)}
$$


Since $F(0,0, x, t)$ is concave in $x$, we have

$$
F(\lambda A+(1-\lambda) B) \geq \lambda F(A)+(1-\lambda) F(B)
$$

for all $\lambda \in[0,1]$ and all points $A=(0,0, x, t), B=(0,0,0, t)$. Since

$$
F(0,0,0, t) \geq 0
$$

we therefore have

$$
\lambda F(0,0, x, t) \leq F(0,0, \lambda x, t)
$$

for all $\lambda \in[0,1]$. Choosing $\lambda=R(t) / u_{\max }(t) \in[0,1]$, we get

$$
\begin{aligned}
\frac{d^{+}}{d t}\left(\frac{u_{\max }(t)}{R(t)}\right) & \leq \frac{u_{\max }(t)}{R^{2}(t)} \cdot\left[\frac{R(t)}{u_{\max }(t)} F\left(0,0, u_{\max }(t), t\right)-F(0,0, R(t), t)\right] \\
& \leq 0 \quad \text { on }[0, T) .
\end{aligned}
$$

The proof of (a) is complete. The proof of (b) is analogous to (a) if we use the inequality

$$
\frac{u_{\min }(t)}{R(t)} F(0,0, R(t), t) \leq F\left(0,0, u_{\min }(t), t\right) .
$$

(c) is an easy consequence of (a) and (b).

Acknowledgments. We would like to thank Ben Andrews for some discussion and the referee for pointing out some minor corrections.

\section{REFERENCES}

[A1] Andrews, B., (1993) Contraction of convex hypersurfaces by their affine normal, J. Diff. Geom. 43, 207-230.

[A2] Andrews, B., (1993) Evolving convex hypersurfaces, Thesis, The Australian National University.

[C] CHow, B., (1995) Geometric aspects of Aleksandrov reflection and gradient estimates for parabolic equations, to appear in Comm. Anal. Geom.

[CG] Chow, B., Gulliver, R., (1996) Aleksandrov reflection and nonlinear evolution equations, I: The $n$-sphere and n-ball, Calc. Var. 4, 249-264.

[CLT] Chow, B., LIOU, L.P., TSAI, D.H., (1996) On the nonlinear parabolic equation $\partial_{t} u=$ $F(\triangle u+n u)$ on $S^{n}$, Comm. Anal. Geom. 4, 415-434.

[CNS] Caffarelli, L., Nirenberg, L., Spruck, J., (1985) The Dirichlet problem for nonlinear second order elliptic equations, III: Functions of the eigenvalues of the Hessian, Acta Math. 155, 261-301.

[CT1] Chow, B., TsaI, D.H., (1996) Geometric expansion of convex plane curves, J. Diff. Geom. 44, 312-330.

[CT2] Chow, B., TSAI, D.H., (1997) Nonhomogeneous Gauss curvature flows, preprint.

[G] Gerhardt, C., (1990) Flow of nonconvex hypersurfaces into spheres, J. Diff. Geom. 32, 299-314.

[GH] Gage, M., Hamilton, R.S., (1986) The heat equation shrinking convex plane curves, J. Diff. Geom. 23, 69-96.

[H] Hamilton, R.S., (1986) Four-manifolds with positive curvature operator, J. Diff. Geom. 24, 153-179.

[Hu] HuISKEN, G., (1988) On the expansion of convex hypersurfaces by the inverse of symmetric curvature functions, unpublised paper. 
[K] KRYLOV, N.V., (1987) Nonlinear elliptic and parabolic equations of the second order, Reidel, Dordrecht.

[KS] Krylov, N.V., Safonov, M.V., (1980) Certain properties of parabolic equations with measurable coefficients, Izv. Akad. Nauk SSSR Ser. Mat. 40, 161-175; English tsansl., Math. USSR Izv. 16 (1981) 151-164.

[T] TsaI, D.H., (1997) Geometric expansion of immersed convex plane curves, preprint.

[TS] Tso, K., (1985) Deforming a hypersurface by its Gauss-Kronecker curvature, Comm. Pure and Appl. Math. 38, 867-882.

[U1] URbas, J.I.E., (1991) An expansion of convex hypersurfaces, J. Diff. Geom. 33, 91-125; (1992) Correction to, ibid. 35, 763-765.

[U2] URBAS, J.I.E., (1990) On the expansion of starshaped hypersurfaces by symmetric functions of their principal curvatures, Math. Zeitscrift 205, 355-3 